\title{
Minimizing Medication Errors by Triangle Check of Look-Alike Sound-Alike Medications from Crash Cart of Gynecology and Obstetrics in Emergency
}

Durr-e-Shahwar Siddiqui, PharmD, MPhil

Supervisor Pharmacy, Al-Khidmat Pharmacy Services, Karachi, Pakistan

\begin{abstract}
Background: According to the Joint Commission, crash cart-related patient safety issues are mixed-up medications, medication errors, damaged or expired medicines, improper checking of crash cart and non-competent staff. The availability of crash cart with all the needed emergency items, appropriate clinical protocols and rapid response team are regarded as important tools for the manage ment of clinical emergencies in obstetrics and gynecology. The aim of this study was to design and implement a strategy to prevent the medication errors of look-alike sound-alike medications in emergency situations from the crash cart of gynecology and obstetrics.

Method: A nurse was assigned to record all the medications-related events in emergency situations along with receiving the medication order from healthcare professionals and dispensing medications from crash cart of gynecology and obstetrics. Triangle check of lookalike sound-alike medications was regarded necessary.

Results: A significant reduction in number of look-alike sound-alike medication errors and near misses was recorded ( $p<0.05$ ).

Discussion: This strategy can be implemented not only to the gynecology and obstetrics but also to other areas of healthcare where look-alike sound-alike medications are stored, dispensed, administered or supplied and will be of great help in emergency situations.

Conclusion: A healthcare professional should be assigned at crash cart to record all the medications-related events and triangle check of dispensed look-alike sound-alike medications must be compulsory in emergency conditions.
\end{abstract}

Keywords: gynecology and obstetrics, crash cart, look-alike sound-alike medications, triangle check

\section{Background}

Promotion of health, prevention from injury and impeding the disease can reduce the frequent occurrence of acute health issues in a healthcare system. Hence, development of emergency medical care guidelines and provision of fundamental emergency medical care can have a marked influence on well-being of patients and can be a life-saving tool (1). According to a bulletin of World Health Organization, a significant number of young patients with critical conditions and mortality were satisfactorily treated by emergency medical care in low middle-income countries. This emphasizes the global importance of emergency medical care in healthcare setups (2). Hence a toolkit was designed by World Health Organization to assess the capacity of healthcare system for crisis management that could strengthen the emergency preparedness of healthcare set-ups ${ }^{(3)}$.

The availability of crash trolley with all the needed emergency items, appropriate clinical protocols, rapid response team which has practiced the emergency drills and can communicate effectively are regarded as important tools for the management of clinical emergencies in obstetrics and gynecology ${ }^{(4)}$. The adverse drug events and medication errors are very common in gynecology and obstetrics but till date very less literature is being published in this regard. A voluntary medication error and

Corresponding author: Durr-e-Shahwar Siddiqui, PharmD, MPhil; Supervisor Pharmacy, Al-Khidmat Pharmacy Services, Karachi, Pakistan

Email: silver shine27@hotmail.com adverse events reporting study in gynecology and obstetrics area of an urban teaching hospital revealed total 82 events in 6 weeks period out of which $56 \%$ events were reported from obstetrics while remaining $44 \%$ were from gynecology ${ }^{(5)}$. Total 1976 medication errors were reported in an observational study conducted upon 10000 women in obstetric emergency ward of a hospital in Egypt in $2010^{(6)}$.

The crash cart must be placed in the emergency areas and must be easily accessible by healthcare professionals. It is recommended to attach a list of all the available items with the crash trolley along with their expiration dates. It is also advised to put the alphabetical list of items on every drawer of the crash trolley so that finding medication in the cart in case of emergency does not become difficult ${ }^{(7)}$. According to the Joint Commission, some of the factors leading to most of the crash cart-related patient safety issues are mixed-up medications, medication errors, damaged or expired medicines, improper checking of crash cart as per policies and procedures and noncompetent staff ${ }^{(8)}$. There must be strengthened system for medicines management and expiry monitoring to prevent any health hazard in a healthcare system ${ }^{(9)}$. Once a medicine is expired, its safety and efficacy becomes uncertain as per U.S. Food and Drug Administration ${ }^{(10)}$.Healthcare professionals must play their role in the monitoring of expired or deteriorated medicines in healthcare areas by following the approved international guidelines of treatment and rationalized usage of medications ${ }^{(11)}$. 
Look-Alike Sound-Alike (LASA) medications are categorized as high-alert medications because they carry an increased risk of causing harm to patient and can also lead to sentinel event when used in error or confused with one another. The Joint Commission suggested that an organization must safely manage its high alert medications and must ensure the safe use of look-alike and sound-alike medications. For this purpose, it was emphasized to develop a high-alert medications list with the help of pharmacy consult as well as to develop and implement a strategy for look-alike sound-alike medications to prevent medication errors. Use of tall-man lettering, identification by stickers, placing look-alike sound-alike medications in separate bins or locations, awareness of all the healthcare professionals for high alert medications, reviewing look-alike sound-alike medications list periodically and displaying look-alike sound-alike medications list in medications rooms, carts, work rooms and treatment rooms are some of the suggestions proposed by The Joint Commission (12).

In 2016, 104 out of 70000 registered medications were identified as look-alike or sound-alike medications in Pakistan according to the report of a local newspaper. 35 out of those 104 look-alike sound-alike medications were thought to cause serious harms to patients if used in confusion or error ${ }^{(13)}$. It is also reported that approximately 400,000 to 500,000 deaths occur every year in Pakistan due to wrong administration of medications or lethal medication errors ${ }^{(14)}$. A triangle check is recommended for look-alike sound-alike medications during dispensing, supply and administration to verify the actual drug against the medication order and label ${ }^{(15)}$.

Three medication errors of look-alike sound-alike medications were reported within a month from the gynecology and obstetrics department of a Secondary Care Hospital at Karachi, Pakistan. These medication errors occurred while using lookalike sound-alike medications from the crash cart of gynecology and obstetrics in emergency situations. Usually a verbal or a written requisition of medication was received from the doctor while using any medication from the crash cart in emergency conditions. The requested medication was dispensed from the crash cart by a nurse and was administered to the patient.
Another nurse documented all the events of medications so as to be replaced in the crash cart as soon as the emergency situation was over. It was a two step process of checking lookalike sound-alike medications which failed in emergency situations leading to medication errors. Although the look-alike sound-alike medications in crash cart were properly labeled and tagged with "CAUTION" label for double-check while dispensing and administering to patient but these precautions were not enough to prevent the medication errors of look-alike soundalike medications in emergency situations.

\section{Objective}

The aim of this study was to design and implement a strategy to prevent the medication errors of look-alike sound-alike medications in emergency situations from the crash cart of gynecology and obstetrics.

\section{Method}

This study was conducted at a Secondary Care Hospital of Karachi, Pakistan for a period of six months. Only look-alike sound-alike medications from the crash cart of gynecology and obstetrics were included in this study. Healthcare professionals consisting of gynecology doctors, nurses and pharmacist were involved in the study. The practices of handling the look-alike sound-alike medications from the crash cart of gynecology and obstetrics were improved by designing and implementing triangle check for emergency situations.

The availability of multiple strengths of a medication was minimized in crash cart. The availability of medications with look-alike packaging or appearance in crash cart was minimized. Tall-man lettering was emphasized for labeling of crash cart medications. Warning labels were pasted not only on the bins but also on the look-alike medications for counterchecking prior administration. Look-alike sound-alike medications were stored far apart from each other in crash cart. Reading the medication labels rather than relying on visual identification of look-alike sound-alike medications was emphasized. Implementation of triangle check for look-alike sound-alike medications was assured. 


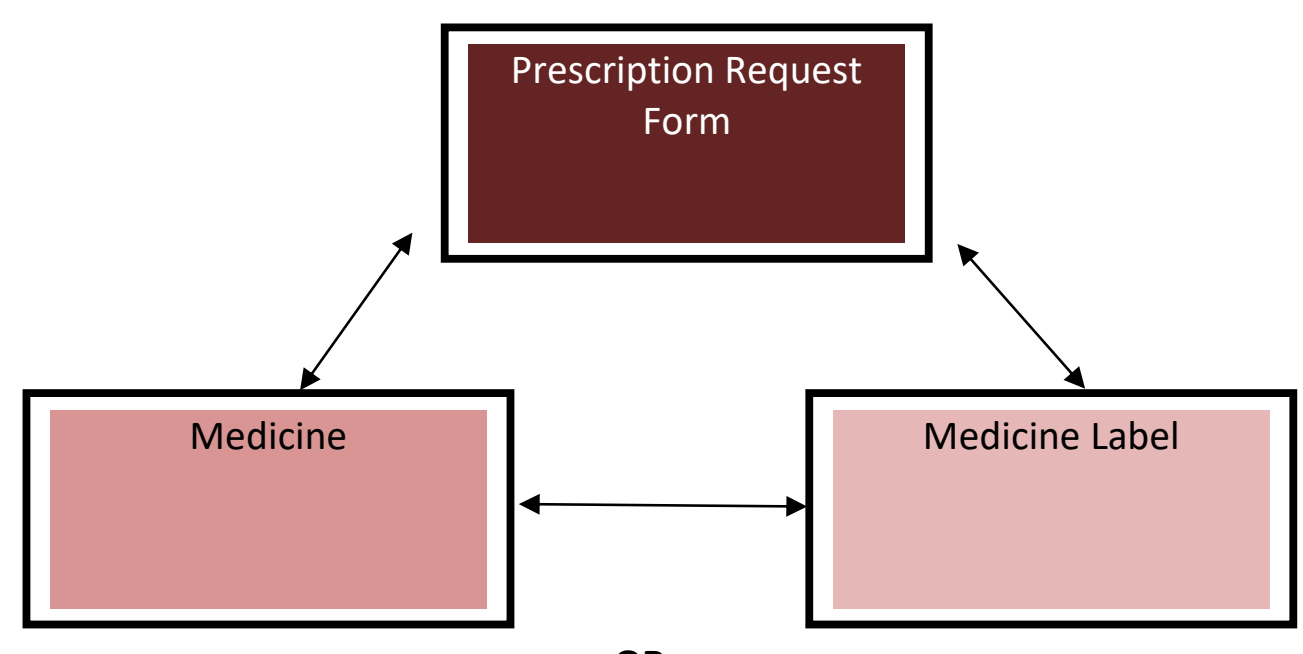

OR

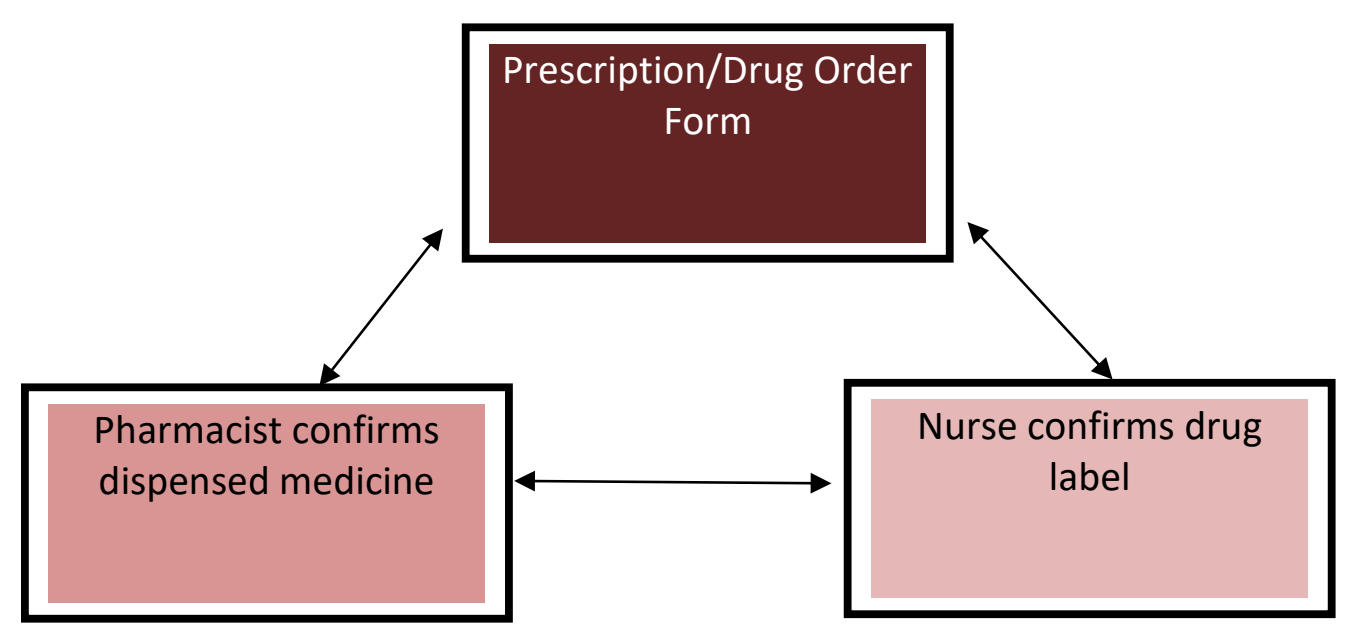

Figure 1: Triangle check of look-alike sound-alike medications suggested by the Pharmaceutical Services Division, Ministry of Health, Malaysia.

\section{Innovation}

A nurse was assigned to record all the medications-related events along with receiving the medication order from doctor or other healthcare professionals and dispensing the medications from crash cart of gynecology and obstetrics in emergency situations. As soon as the medication order is received, nurse at the crash cart read the order carefully and dispensed the required medication to another nurse. The label of dispensed medicine from the crash cart was counterchecked by the medication receiving nurse. A third check of the dispensed medicine was performed by the medicine ordering healthcare professional before he/she administered it to the patient. 


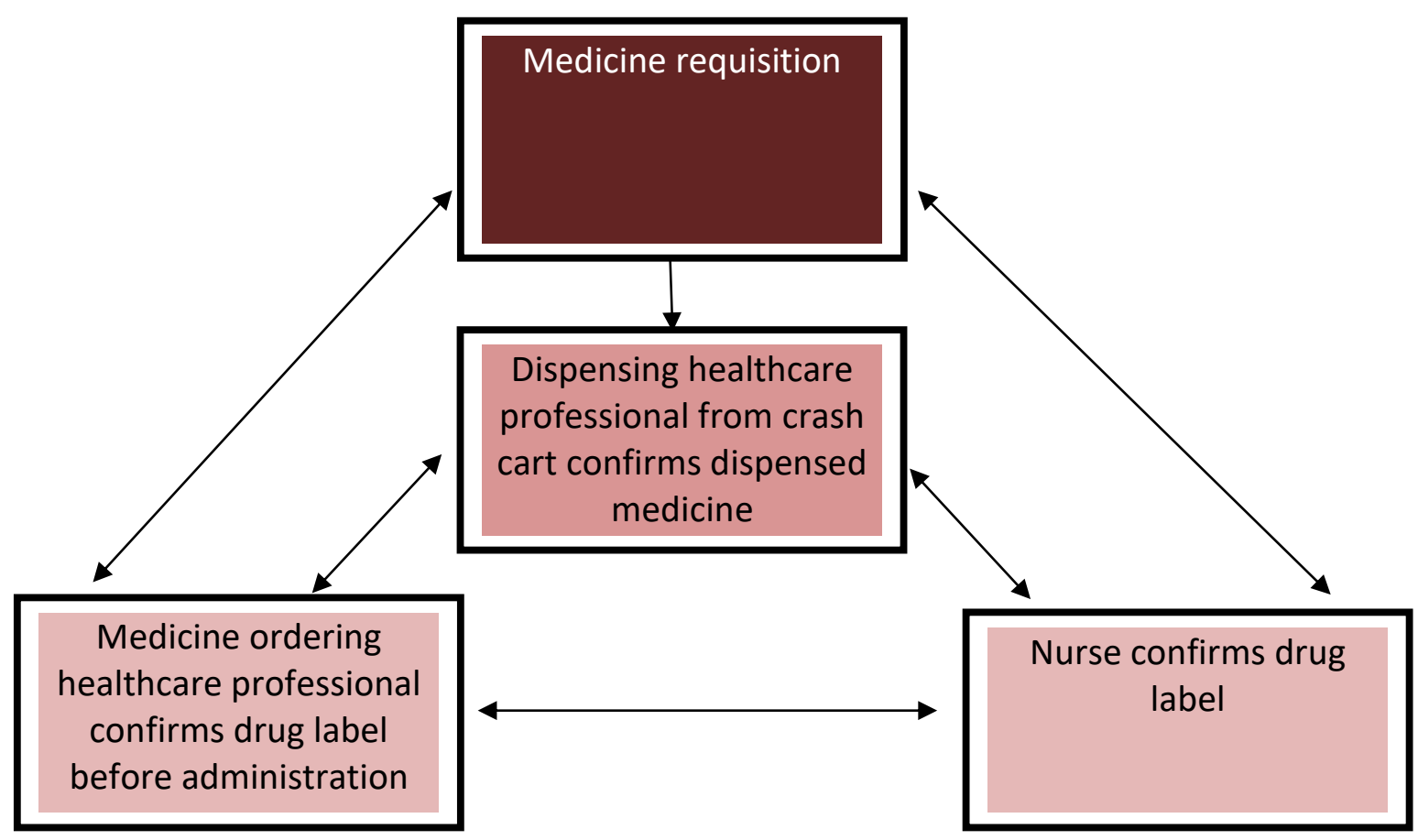

Figure 2: The innovated setup for triangle check of look-alike sound-alike medications from crash trolley of gynecology and obstetrics by healthcare professionals to avoid medication errors in emergency situations.

The triangle check of look-alike sound-alike medications dispensed from crash cart of gynecology and obstetrics was assured even in the absence of Pharmacist in emergency situations because this innovation involved two nurses and a doctor for triangle check. The separate nurse assigned at crash cart in emergency conditions helped in ensuring a triangle check in emergency conditions.

\section{Results}

A total of 367 patients were included in this study within a period of 6 months. All the patients were pregnant and were admitted in gynecology and obstetrics department. Of these 367 patients, 231 patients had caesarian section, 129 patients had spontaneous vaginal delivery while 7 patients had gone through dilation and evacuation procedure. The study was conducted at a Secondary Care Hospital of Karachi, Pakistan.

Total 13 medications were recognized as look-alike sound-alike medications in the crash cart of gynecology and obstetrics (see Table 1). The list of those look-alike sound-alike medications was pasted visibly near the crash cart. 
Table 1: List of Look-alike Sound-alike Medications with Medication Errors and Near Misses

\begin{tabular}{|c|c|c|c|c|c|}
\hline \multicolumn{4}{|c|}{$\begin{array}{l}\text { Look-alike Sound-alike Medications in Crash } \\
\text { Cart of Gynecology and Obstetrics }\end{array}$} & \multirow{2}{*}{$\begin{array}{c}\text { Medication } \\
\text { Errors }\end{array}$} & \multirow{2}{*}{$\begin{array}{c}\text { Near Misses } \\
n=4\end{array}$} \\
\hline Inj. Atropine & Inj. Vitamin K & Inj. Adrenaline & $\begin{array}{l}\text { Inj. } \\
\text { Furosemide }\end{array}$ & & \\
\hline Inj. Ketamine & $\begin{array}{l}\text { Inj. Bupivacaine } \\
\text { spinal } 0.5 \%\end{array}$ & & & $\mathrm{n}=3$ & $\mathrm{n}=0$ \\
\hline $\begin{array}{l}\text { Potassium chloride } \\
25 \mathrm{ml} \text { ampoule }\end{array}$ & $\begin{array}{l}0.9 \% \text { Normal Saline } \\
25 \mathrm{ml} \text { ampoule }\end{array}$ & $\begin{array}{l}25 \% \text { Dextrose } \\
25 \mathrm{ml} \text { ampoule }\end{array}$ & & $\mathrm{n}=0$ & $\mathrm{n}=1$ \\
\hline $\begin{array}{l}\text { Inj. Tranexamic acid } \\
\text { 500mg }\end{array}$ & $\begin{array}{l}\text { Inj. Diclofenac } \\
\text { sodium }\end{array}$ & & & $\mathrm{n}=0$ & $\mathrm{n}=1$ \\
\hline Inj. Oxytocin & $\begin{array}{l}\text { Inj. Nalbuphine } \\
\mathrm{HCl} 10 \mathrm{mg}\end{array}$ & & & $\mathrm{n}=0$ & $\mathrm{n}=0$ \\
\hline
\end{tabular}

Table 2: Reported number of Medication Errors and Near Misses of Look-alike Sound-alike Medications within Six Months

\begin{tabular}{|l|c|c|c|}
\hline \multicolumn{1}{|c|}{ Month } & Study Population & $\begin{array}{c}\text { Number of Look-alike Sound- } \\
\text { alike Medication Errors }\end{array}$ & $\begin{array}{c}\text { Number of Near Misses } \\
\text { of Look-alike Sound-alike } \\
\text { Medications }\end{array}$ \\
\hline April & $\mathrm{n}=46$ & 3 & 2 \\
\hline May & $\mathrm{n}=42$ & 1 & 2 \\
\hline June & $\mathrm{n}=52$ & 0 & 1 \\
\hline July & $\mathrm{n}=75$ & 0 & 1 \\
\hline August & $\mathrm{n}=69$ & 0 & 0 \\
\hline September & $\mathrm{n}=56$ & 0 & 0 \\
\hline
\end{tabular}

A significant reduction in number of look-alike sound-alike medication errors was reported in the $1^{\text {st }}$ month after implementing triangle check of look-alike sound-alike medications in the gynecology and obstetrics for emergency situations $(p<0.05)$. Reduction was very much significant in $2^{\text {nd }}$, $3^{\text {rd }}, 4^{\text {th }}$ and $5^{\text {th }}$ month of ensuring triangle check of look-alike sound-alike medications in emergency situations at gynecology and obstetrics. Number of near misses also reduced significantly with time $(p<0.05)$ because the medication errors of look-alike sound-alike medications that were about to occur were identified during triangle check stages (see Table 2).

\section{Discussion}

For the management of clinical emergencies in gynecology and obstetrics, a crash trolley with all the needed emergency items is necessary. Moreover the team of healthcare professionals must be well-aware of the clinical protocols and must follow the patient safety guidelines because medication errors and adverse drug reactions are very common in gynecology and obstetrics. Wrong medicine, incorrect dose, wrong frequency or wrong timing of medication and non-implementation of medication order details are some of the medication errors occurring most frequently during labor and delivery (16). An urban teaching hospital reported total 82 such events from gynecology and obstetrics in 6 weeks period. It is emphasized that the healthcare professionals and other hospital staff in obstetrics must know that medication errors cause severe harm to the patients and must take precautionary measures (17). These precautionary strategies must include defense mechanisms, reduction of complexity and reminders of safety measures at patient care stations ${ }^{(18)}$. As per The Joint Commission, mixed-up medications, medication errors, damaged or expired medicines, improper checking of crash cart as per policies and procedures and non-competent staff are some of the factors leading to most of the crash cart-related patient safety issues. Hence it is suggested that an organization must safely manage its high alert medications and must ensure the safe use of look-alike and sound-alike medications.

Three medication errors occurred in a Secondary Care Hospital of Karachi, Pakistan, while using look-alike sound-alike medications from the crash cart of gynecology and obstetrics in emergency situations despite proper label and "CAUTION" tag for double-check of look-alike sound-alike medications. So this study was aimed to formulate and implement strategies to prevent the medication errors of look-alike sound-alike medications in emergency situations from the crash cart of gynecology and obstetrics. The study included 367 pregnant patients admitted in hospital and was conducted for 6 months. The availability of multiple strengths or look-alike packaging of 
a medication was minimized in crash cart. Look-alike soundalike medications were stored distantly in cart with tall-man lettering and warning labels were pasted on medications as well as on bins. Triangle check of look-alike sound-alike medications was implemented and assured even in emergency situations. The suggested model of triangle check of look-alike sound-alike medications by the Pharmaceutical Services Division, Ministry of Health, Malaysia focused on prescription or medication requisition to be received and medicine must be checked against the label. Nurse counterchecks the medication against label prior administration. Prescription is generally received at Pharmacy and Pharmacist counterchecks the medication against the requisition but this process could not be followed in emergency situations at gynecology and obstetrics because most of the received medication orders in such situations are verbal and pharmacist is also not assigned during gynecological procedures.

So, a nurse was assigned to record all the medications-related events along with receiving the medication order from doctor or other healthcare professionals because the verbal medication orders must be documented by a healthcare professional so that the prescriber could countercheck it for medication accuracy review ${ }^{(19)}$. This assigned nurse dispensed the medications from crash cart of gynecology and obstetrics in emergency situations. Nurse at the crash cart listened or read the order (verbal/written) carefully when medication order was received and dispensed the required medication to another nurse. The label of dispensed medicine from the crash cart was counterchecked by the medication receiving nurse. A third check of the dispensed medicine was performed by the medicine ordering healthcare professional before he/she administered it to the patient. This innovation caused significant reduction in look-alike sound-alike medication errors and near misses $(p<0.05)$ within six months.

This strategy can be implemented not only to the gynecology and obstetrics but also to other areas of healthcare where lookalike sound-alike medications are stored, dispensed, administered or supplied and can help even in emergency situations. Strict collaboration between healthcare professionals and awareness about look-alike sound-alike medications is necessary.

Healthcare organizations in the World will be highly benefitted by the implementation of this suggested triangle check of lookalike sound-alike medications as a risk management strategy for patient safety. A healthcare professional preferably a nurse must be appointed as a medication safety staff for the usability of triangle check of look-alike sound-alike medications approach. The medication safety staff will be responsible for the triangle check of all look-alike sound-alike medications before their administration to patient in emergency situations. Moreover, the medication safety staff will also be responsible for the proper storage and expiry checking of look-alike soundalike medications in non-emergency conditions throughout the healthcare organization.

\section{Conclusion}

Triangle check of look-alike sound-alike medications of crash cart must be performed in emergency situations to prevent medication errors in gynecology and obstetrics and healthcare professionals must be well-aware of look-alike sound-alike medications and triangle check strategy for patient safety. A healthcare professional should be assigned at crash cart to record all the medications-related events and countercheck of dispensed look-alike sound-alike medications in emergency against medication order.

Conflicts of interest: The author has no conflicts of interest to report.

\section{References}

1. Razzaq J A and Kellermann A L. Emergency medical care in developing countries: is it worthwhile?, Policy and Practice, Bull World Health Organ, 2002; 80 (11): 900-5

2. Obermeyer Z, Abujaber S, Makar M, Stoll S, Kayden S $R$, Wallis L A and Reynolds T A. Emergency care in 59 low- and middle-income countries: a systematic review, Bull World Health Organ, 2015; 93: 577-586

3. World Health Organization, Toolkit for assessing health-system capacity for crisis management, Available from: http://www.euro.who.int/_data/assets/pdf_file/000 8/157886/e96187.pdf

4. Committee Opinion, Preparing for Clinical Emergencies in Obstetrics and Gynecology, The American College of Obstetricians and Gynecologists, March 2014; 590

5. November M, Chie L and Weingart S N. PhysicianReported Adverse Events and Medical Errors in Obstetrics and Gynecology, Advances in Patient Safety: New Directions and Alternative Approaches, Aug 2008; 1

6. Kandil M, Sayyed T, Emarh H, Ellakwa H and Masood A. Medication errors in the obstetrics emergency ward in a low resource setting, J Matern Fetal Neonatal Med, Aug 2012; 25 (8): 1379-82

7. Jacquet $G$ A, Hamade B, Diab K A, Sawaya R, Dagher G $A$, Hitti E and Bayram J D. The Emergency Department Crash Cart: A systematic review and suggested contents, World J Emerg Med, 2018; 9 (2):93-98

8. The Joint Commission. Crash-cart preparedness, Quick Safety, April 2017; 32:2 
9. Kamba P F, Ireeta M E, Balikuna S and Kaggwa B. Threats posed by stockpiles of expired pharmaceuticals in low- and middle -income countries: a Ugandan perspective, Bull World Health Organ, Aug 2017; 95 (8):594-598

10. U.S. Food and Drug Administration. Don't Be Tempted to Use Expired Medicines, FDA, 2016; Available from: https://www.fda.gov/drugs/specialfeatures/dont-be-tempted-use-expired-medicines

11. Ebrahim A J, Teni F S and Yimenu D K. Unused and Expired Medications: Are They a Threat? A FacilityBased Cross-Sectional Study, J Prim Care Community Health, 2019; 10

12. The Joint Commission. Managing HighAlert/Hazardous and Look-Alike-Sound-Alike Medications in Ambulatory Care Settings, Aug 2020; Available from:

https://www.jointcommission.org/resources/newsand-multimedia/blogs/ambulatorybuzz/2020/01/08/managing-high-alert-hazardousand-look--alike-sound-alike-medications-inambulatory-care-settings/

13. The News. 104 medicines in Pakistan identified as 'look-alike, sound-alike', Dec 2016; Available from: https://www.thenews.com.pk/print/172599-104medicines-in-Pakistan-identified-as-look-alike-soundalike
14. Dawn. Medication errors cause half a million deaths in Pakistan, say pharmacists, Oct 2017; Available from:

https://www.dawn.com/news/1362951\#: :text=\%E2 \%80\%9CWe\%20don't\%20have\%20actual,attention\%2 0of\%20authorities\%20and\%20pharmacists.\%E2\%80\% 9D

15. Guide on handling look alike sound alike medications. Pharmaceutical Services Division, Ministry of Health, Malaysia. (First edition) 2012

16. Smith JG, Mertz HL, Merrill DC. Identifying Risk Factors for Uterine Rupture, Survey of Anesthesiology, Feb 2009; 53 (1):17-18

17. Kfuri TA, Morlock L, Hicks RW, Shore AD. Medication Errors in Obstetrics, Clinics in Perinatology, Mar 2008; 35(1):101-117

18. Gluck PA. Medical Error Theory, Obstetrics and Gynecology Clinics of North America, Mar 2008; 35(1):11-17

19. Patient Safety in Obstetrics and Gynecology, The American College of Obstetricians and Gynecologists, Dec 2009; Committee Opinion, 447, Available from: https://www.acog.org/clinical/clinicalguidance/committeeopinion/articles/2009/12/patient-safety-inobstetrics-and-gynecology 